\title{
It takes two to tango: mast cell and Schwann cell interactions in neurofibromas
}

\author{
David H. Viskochil \\ Department of Pediatrics, School of Medicine, University of Utah, \\ Salt Lake City, Utah, USA
}

\begin{abstract}
Neurofibromas are benign tumors comprised primarily of Schwann cells and fibroblasts. Mast cell infiltration is a well-known phenomenon; however, their role in tumor pathogenesis has been enigmatic. In an elegant set of experiments using cells derived from a murine model of neurofibromatosis 1 (NF1), Yang et al. (see the related article beginning on page 1851) dissect the molecular pathways involved in mast cell migration to neurofibromin-deficient Schwann cells. These results set the stage for rational development of therapeutics that could influence the multicellular microenvironment of neurofibromas to inhibit the development and/or progression of these tumors in human NF1.
\end{abstract}

J. Clin. Invest. 112:1791-1793 (2003). doi:10.1172/JCI200320503.

In an Original article series published through the March of Dimes Birth Defects Foundation in 1981, Vic Riccardi outlined his "NF cellular interaction hypothesis," implicating the mast cell as a major player neurofibroma formation (1). He was attempting to explain the variable expression of neurofibromatosis 1 (NF1) by cellular interactions and the finding of high numbers of mast cells in neurofibromas when he posited that "the mast cell now is seen not as a secondary arrival in a developing neurofibroma but as an inciting factor contributing in a primary, direct fashion to tumor development." It has taken over 20 years, but in this issue of the JCI, Yang et al. (2) clearly set the record straight by identifying the molecular mechanisms underlying mast cell infiltration of neurofibromas. The authors demonstrated that the inciting factor for mast cell migration is Kit ligand and that it is hypersecreted from $\mathrm{Nf1}^{-/-}$ Schwann cell populations. Nevertheless, that Riccardi hypothesized para-

\footnotetext{
Address correspondence to: David H. Viskochil, 50 North Medical Drive, Room 2C412, School of Medicine, University of Utah, Salt Lake City, Utah 84112, USA. Phone: (801) 581-8943; Fax: (801) 585-7252; E-mail: DAVE.VISKOCHIL@hsc.utah.edu.

Conflict of interest: The author has declared that no conflict of interest exists.

Nonstandard abbreviations used: neurofibromatosis 1 (NF1); malignant peripheral nerve sheath tumor (MPNST).
}

crine events involving the mast cell as a major player in neurofibroma formation is a testament to his intuition regarding the pathogenesis of this complex genetic condition.

Yang and colleagues were intrigued by the seminal observations of Zhu et al. (3) of neurofibroma formation in mice expressing the conditional deletion of Nf1, whereby double inactivation of the gene $\mathrm{Nf1}^{-/-}$in Schwann cells could only induce neurofibroma formation in the context of a heterozygous $\mathrm{Nf1}^{+/-}$genetic background. This supports the paracrine model for neurofibromas; other cells in these mixed-cell tumors must be haploinsufficient for neurofibromin to support proliferation. Yang et al. (2) have taken these observations a step further by dissecting the cell types and the molecular mechanisms by which the tumor microenvironment contributes to neurofibroma formation. Through a set of elegant experiments, they have delineated a complex biological system and provided a model demonstrating not just who the major players are in this process, but how those players interact in concert to develop neurofibromas (Figure 1).

\section{Neurofibroma microenvironment}

The basic components of an evolving neurofibroma are: (i) $\mathrm{Nf1}^{-/-}$Schwann cells, which act as tumorigenic instigators (4-7); (ii) $\mathrm{Nf1}^{+/-}$mast cells, which act as inducers (2); and (iii) $\mathrm{Nf1}^{+/}$- fibroblasts, Schwann cells, perineural cells, and endothelial cells, which act as the responders (8). The pathways involved in neurofibroma formation identified by Yang et al. (2) involve Kit ligand (secreted by $\mathrm{Nf1}^{-/}$ Schwann cells), c-Kit receptor (expressed by mast cells), $\alpha 4 \beta 1$ integrin (a mast cell surface protein), VCAM-1 (an endothelial receptor for $\alpha 4 \beta 1$ integrin), Ras (a substrate for neurofibromin), and the class $\mathrm{I}_{\mathrm{A}}$-PI3KRac2 pathway (mast cell transduction downstream of activated Ras). The primary observation of Yang et al. is the enhanced migration of neurofibromin haploinsufficient mast cells toward the double-inactivated, $\mathrm{Nf1}^{-/}$ Schwann cells that secrete five times the normal levels of Kit ligand. According to this paradigm, in human neurofibromas, a "second hit," or somatic mutation of the normal NF1 tumor suppressor gene allele in heterozygous Schwann cells as a random event, is the initiating event which sets the stage for mast cells to induce neurofibroma formation by paracrine/ autocrine mechanisms.

\section{Mast cells and neurofibromas}

The presence of mast cells in human neurofibromas, initially reported in 1911 by Greggio (9), is well known to clinical pathologists. Additional studies have since supported the finding of mast cells in peripheral nerve trunks $(10,11)$, peripheral nerve tumors (12-14), and neurofibromas in neurofibromatosis $1(15-17)$. The potential involvement of the c-Kit receptor and its ligand $(18,19)$ in the formation of neurofibromas has also been reported, but Yang et al. (2) are the first investigators to evaluate living cells of different genetic backgrounds in order to demonstrate how increased mast cells populate neurofibromas. Now that a plausible mechanism has been established for the migration of mast cells to developing neurofibromas, it is important to dissect potential mechanisms whereby mast cells can induce cells in the microenvironment to either tolerate or enhance cell proliferation. Because we know that neurofibromas, like other tumors, are complex tissues 


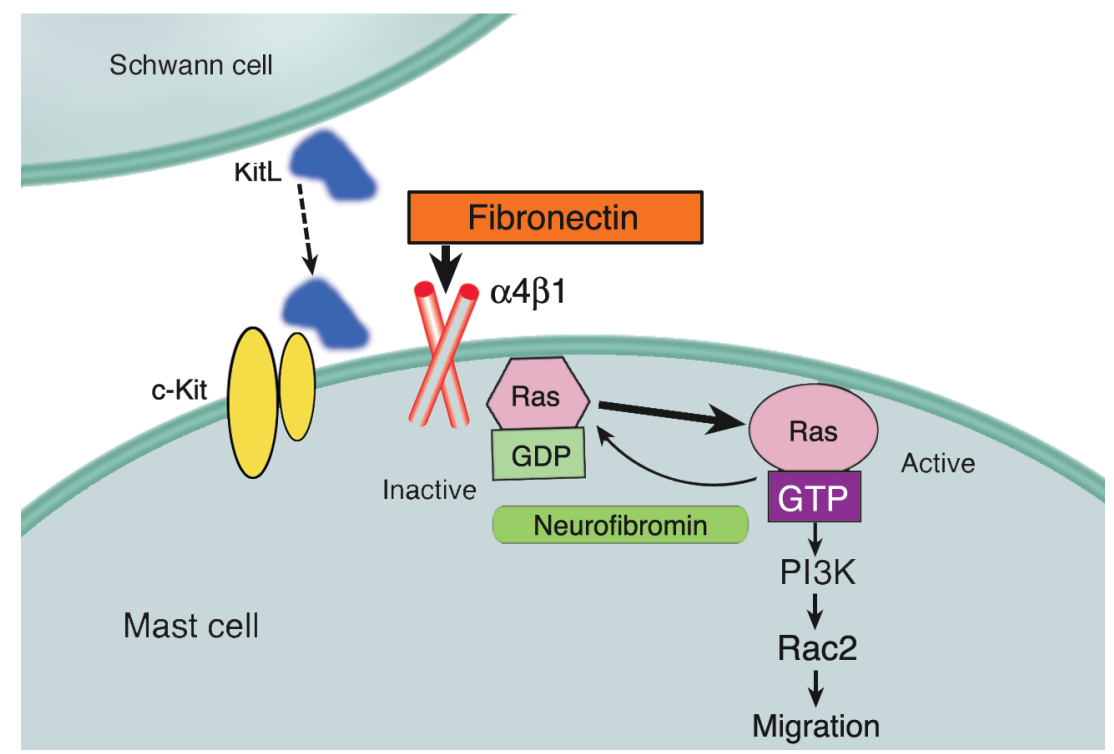

\section{Figure 1}

Illustration depicting the molecular pathways involved in mast cell recruitment to neurofibromas. A neurofibromin-deficient Schwann cell $\left(\mathrm{Nf1}^{--}\right)$secretes five times the normal Kit ligand, which serves as a chemoattractant for mast cells expressing c-Kit. Mast cell migration is mediated by the Ras/PI3K/Rac2 signal transduction pathway, which is enhanced in $\mathrm{Nf1}^{+/-}$cells. Although not shown, endothelial cells also play a role in mast cell migration through the interaction of the endothelial cell VCAM-1 receptor and $\alpha 4 \beta 1$ integrin of the mast cell. Heterozygous inactivation of $N f 1$ promotes rapid mast cell haptotaxis specifically on $\alpha 4 \beta 1$ integrins in response to KitL. It is known that mast cells are activated in neurofibromas and degranulate; however it is yet to be determined if and how the presence of mast cells induces tumor progression. Figure courtesy of D. Wade Clapp, Indiana University School of Medicine. KitL, Kit ligand.

with interactions between genetically altered Schwann cells, mast cells, and other supporting coconspirators lead to cancer development (20), it now becomes important to determine the role mast cells play in the microenvironment of NF1-related peripheral nerve sheath tumors.

\section{Mast cells in cancer}

Mast cell secretions are known to be integral components of wound healing and tissue repair, even in the peripheral nervous system. In addition to their role in inflammation, mast cells provide mitogens for fibroblasts, endothelial cells, and nerve cells to enhance tissue remodeling, and they are also now being recognized as potential epigenetic contributors to cancer (21). It will be important to establish the functions of the mast cell after it has migrated to the Kit ligand-secreting Schwann cells. In addition to secreting mitogenic and angiogenic substances, including basic FGF, VEGF, histamine, heparin, prostaglandins, leu- the early treatment and/or prevention of neurofibromas.

\section{Mast cells: missing in action}

There is one caveat to bear in mind in regard to the role $\mathrm{Nf1}^{+/-}$mast cells may play as they nestle into their newfound microenvironment. With respect to the role of inflammation in cancer and the recognition that mast cells are one of the inflammatory cell types involved in protumor actions (26), it should be acknowledged that neurofibromas are benign tumors, especially the dermal neurofibromas, which never progress to malignancy. It is also important to recognize that the neurofibromas that develop in the mouse models are not dermal neurofibromas, but are more like human plexiform neurofibromas. These tumors often progress to malignant peripheral nerve sheath tumors (MPNSTs); however, unlike in other tumor model systems where inflammatory cells are in excess in tumor microenvironments, there are relatively few mast cells in MPNSTs (ref. 27; H. Zhou, personal communication). Is it possible that an influx of mast cells, drawn by a small cohort of malignant $\mathrm{Nf1}^{-/-}$Schwann cells, induces fibroblasts to synthesize the plethora of extracellular matrix seen in dermal neurofibromas, which could then serve as a "glue" to corral the deviant Schwann cells? By this reasoning, only when mast cells are lost, as seen in the MPNSTs, would there be diminution of the anti-cancer effects of mast cells in maintaining the benign nature of neurofibromas. Thus, until the paracrine/autocrine paradigm is deciphered in neurofibroma formation, it behooves the NF1 research community to continue working toward the identification of mast cell signals (inducers) that specify responses from the microenvironment before implementing clinical trials using agents that inhibit mast cell migration toward the tumorigenic Schwann cell. To this end, Yang and colleagues (2) will likely make more outstanding contributions both to the NF1 research field and to the work of those who are dissecting the roles of inflammation and the microenvironment in cancer. 
1. Riccardi, V.M. 1981. Cutaneous manifestation of neurofibromatosis: Cellular interaction, pigmentation, and mast cells. In Birth defects: original article series. Volume 17. R. Blandau, N. Paul, and F. Dickman, editors. Alan R. Lis Inc. New York, New York, USA. 129-145.

2. Yang, F.-C., et al. 2003. Neurofibromin-deficient Schwann cells secrete a potent migratory stimulus for $\mathrm{Nf}^{+/-}$mast cells. J. Clin. Invest. 112:1851-1861. doi:10.1172/JCI200319195.

3. Zhu, Y., Ghosh, P., Charnay, P., Burns, D.K., and Parada, L.F. 2002. Neurofibromas in NF1: Schwann cell origin and role of tumor environment. Science. 296:920-922.

4. Kluwe, L., Friedrich, R., and Mautner, V.F. 1999. Loss of NF1 allele in Schwann cells but not in fibroblasts derived from an NF1-associated neurofibroma. Genes Chromosomes Cancer. 24:283-285.

5. Serra, E., et al. 2000. Schwann cells harbor the somatic NF1 mutation in neurofibromas: evidence of two different Schwann cell subpopulations. Hum. Mol. Genet. 12:3055-3064.

6. Rutkowski, J.L., Wu, K., Gutmann, D.H., Boyer, P.J., and Legius, E. 2000. Genetic and cellular defects contributing to benign tumor formation in neurofibromatosis type 1 . Hum. Mol. Genet. 12:1059-1066.

7. Muir, D., Neubauer, D., Lim, I.T., Yachnis, A.T., and Wallace, M.R. 2001. Tumorigenic properties of neurofibromin-deficient neurofibroma Schwann cells. Am. J. Pathol. 158:501-513.
8. Fialkow, P.J., Sagebiel, R.W., Gartler, S.M., and Rimoin, D.L. 1971. Multiple cell origin of here ditary neurofibromas. N. Engl. J. Med. 284:298-300.

9. Greggio, H. 1911. Les cellules granuleuses (Mastzellen) dans les tissus normaux et dans certaines maladies chirurgicales. Arch. Med. Exp. 23:323-375

10. Gamble, H.J., and Goldby, S. 1961. Mast cells in peripheral nerve trunks. Nature. 189:766-767.

11. Olsson, Y. 1971. Mast cells in human peripheral nerve. Acta Neurol. Scand. 47:357-368.

12. Baroni, C. 1964. On the relationship of mast cells to various soft tissue tumours. $\mathrm{Br}$. J. Cancer. 18:686-690.

13. Pineda, A. 1965. Mast cells - their presence and ultrastructural characteristics in peripheral nerve tumors. Arch. Neurol. 13:372-382.

14. Isaacson, P. 1976. Mast cells in benign nerve sheath tumours. J. Pathol. 119:193-196.

15. Giorno, R., Lieber, J., and Claman, H.N. 1989. Ultrastructural evidence for mast cell activation in a case of neurofibromatosis. Neurofibromatosis. 2:35-41.

16. Carr, N.J., and Warren, A.Y. 1993. Mast cell numbers in melanocytic naevi and cutaneous neurofibromas. J. Clin. Pathol. 46:86-87.

17. Nurnberger, M., and Moll, I. 1994. Semiquantitative aspects of mast cells in normal skin and in neurofibromas of neurofibromatosis types 1 and 5. Dermatology. 188:296-299.

18. Hirota, S., et al. 1993. Possible involvement of c-kit receptor and its ligand in increase of mast cells in neurofibroma tissues. Arch. Pathol. Lab. Med. 117:996-999.

19. Bedache, A., Muja, N., and De Vries, G.H. 1998. Expression of Kit in neurofibromain-deficien human Schwann cells: role in Schwann cell hyperplasia associated with type 1 neurofibromatosis. Oncogene. 17:795-800.

20. Hannahan, D., and Weinberg, R.A. 2000. The hallmarks of cancer. Cell. 100:57-70.

21. Coussens, L.M., and Werb, Z. 2001. Inflammatory cells and cancer: think different. J. Exp. Med. 193:F23-F26.

22. Greenberg, G., and Burnstock, G. 1983. A novel cell-to-cell interaction between mast cells and other cell types. Exp. Cell. Res. 147:1-13.

23. Riccardi, V.M. 1987. Mast-cell stabilization to decrease neurofibroma growth. Arch. Dermatol. 123:1011-1016.

24. Riccardi, V.M. 1993. A controlled multiphase trial of ketotifen to minimize neurofibroma-associated pain and itching. Arch. Dermatol. 129:577-581.

25. Meyer, L.J. 1993. Drug therapy for neurofibromatosis? Arch. Dermatol. 129:625-626.

26. Coussens, L.M., and Werb, Z. 2002. Inflammation and cancer. Nature. 420:860-867.

27. Johnson, M.D., Kamso-Pratt, J., Federspiel, C.F. and Whetsell, W.O. 1989. Mast cell and lymphoreticular infiltrates in neurofibromas. Comparison with nerve sheath tumors. Arch. Pathol. Lab. Med. 113:1263-1270.

\title{
Tumbling down a different pathway to genetic instability
}

\section{Haiwei H. Guo and Lawrence A. Loeb \\ Joseph Gottstein Cancer Research Laboratory, Department of Pathology, University of Washington, Seattle, Washington, USA}

\begin{abstract}
Ulcerative colitis (UC), a chronic inflammatory condition associated with a predisposition to colon cancer, is frequently characterized by DNA damage in the form of microsatellite instability (MSI). A new report links inflammation in UC with increases in the DNA repair enzymes 3-methyladenine DNA glycosylase and apurinic/apyrimidinic endonuclease, and, paradoxically, with increased MSI (see the related article beginning on page 1887). These findings may represent a novel mechanism contributing to MSI in chronic inflammation.
\end{abstract}

J. Clin. Invest. 112:1793-1795 (2003). doi:10.1172/JCI200320502.

Address correspondence to: Lawrence A. Loeb, Department of Pathology, K-072 HSB, 1959 NE Pacific Street, University of Washington, Seattle, Washington 98195-7705, USA. Phone: (206) 543-6015; Fax: (206) 543-3967; E-mail: laloeb@u.washington.edu. Conflict of interest: The authors have declared that no conflict of interest exists. Nonstandard abbreviations used: ulcerative colitis (UC); chromosomal instability (CI); microsatellite instability (MSI); base excision repair (BER); 3-methyladenine DNA glycosylase (AAG); apurinic/apyrimidinic endonuclease (APE1); apurinic/apyrimidinic (AP); reactive oxygen species (ROS); polymerase $\beta$ (pol $\beta$ ).

\section{Ulcerative colitis, genetic instability, and cancer}

A longstanding question in cancer research is the strong association between certain chronic inflammatory conditions and the concomitant elevated risk for malignancy in affected tissues. Understanding the molecular mechanisms driving the progression to cancer may not only provide more effective means of prevention but also shed light on mechanisms of carcinogenesis. Ulcerative colitis (UC), which affects as many as 6 per 100,000 people in the
United States, is a relapsing form of chronic inflammatory disease of the large bowel. Patients with more than a 10-year history of disease have a 20- to 30 -fold greater risk of developing colorectal cancer (1). Both chromosomal instability (CI) and microsatellite (short, repetitive nucleotide sequences in DNA) instability (MSI) are present in UC and can be detected early in dysplastic, premalignant tissues $(2,3)$. What are the sources of these changes in DNA sequence? Chromosomal changes are frequent in cancer and MSI has been clearly documented as a result of mutations in mismatch repair enzymes in the hereditary nonpolyposis colon cancer syndrome. MSI is also observed in many other malignancies (4). Accumulation of mutations in microsatellites could be the result of alterations in enzymes that normally guarantee DNA stability, thus leading to a mutator phenotype (4). Existing hypotheses postulate that excess amounts of free radicals found in inflamed UC tissues overwhelm DNA repair pathways, leading to the accumulation of damaged DNA (5), or that mismatch repair pathways are inactivated, either directly by oxidative stress (6) or by hypermethylation (7). In the traditional view, members of DNA repair pathways are heroic players, stoically laboring against the overwhelming tide of genetic insults thrown their way. 